\title{
Aus den Papieren Constantin von Monakows (1853-1930)
}

\author{
Von Alfred W. Gubser
}

Die nachfolgenden Ausführungen sollen den berühmten Schweizer russischer Abkunft, Constantin von Monakow, etwas näher bringen. Er war Neurologe, Gründer der schweizerischen, Mitgründer der deutschen neurologischen Gesellschaft, Professor und erster Direktor des hirnanatamischen Institutes in Zürich.

Anhand des Manuskripts der hoffentlich bald zur Veröffentlichung gelangenden Selbstbiographie - der Vita mea, wie er sie nannte - (die von Professor E.H. Ackerknecht und vom Schreibenden bearbeitet werden) und von Briefen aus der Sammlung des medizinhistorischen Institutes, soll diese eigenwillige Persönlichkeit durch Schilderung einiger typischer Lebenssituationen (Studium, Dissertation, Habilitation, Professur) beleuchtet werden. Die Situation der Neurologie jener Zeit ganz allgemein darzulegen, würde zu weit führen, von den schweizerischen Verhältnissen sei mit einigen Namen nur die Situation Zürichs in Erinnerung gerufen.

So waren es Goll (als Leiter der medizinischen Poliklinik), Griesinger (als Direktor der medizinischen Klinik), Bernhard von Gudden (als Professor für Psychiatrie), Huguenin (als weiterer Inhaber des internistischen Lehrstuhls), Hitzig und Forel (als Psychiatrieprofessoren im Hauptfach), die sich alle nebenbei mit der Neurologie befaßten.

Dies zu erwähnen ist nicht unwichtig zum besseren Verständnis der späteren Schwierigkeiten, die von Monakow hatte bei der Schaffung des Lehrstuhls und der damit verbundenen erstmaligen Abtrennung seines Faches von der inneren Medizin.

Nach der in Rußland (Wologda) verlebten Jugendzeit kam Constantin mit 14 Jahren nach Zürich, und bereits der Wechsel von der vom Vater gewollten Privatschule zum öffentlichen Gymnasium weist typische Charakterzüge auf: Es war ihm verleidet die Privatschule zu besuchen, und so schrieb er ein Aufnahmegesuch, unterschrieb es mit seinem Namen und dem unwahren Zusatz: Im Namen meines Vaters, obwohl dieser von der Angelegenheit keine Ahnung hatte. Das Resultat der Aufnahmeprüfung kam dann aber in Vaters Hände. Dieser, Ivan von Monakow, war in seiner ganzen Haltung ein unnahbarer, eigener und vornehmer Herr. Seine Schlußverfügung lautete: «Hast du es fertig gebracht, deine weitere Erziehung und 
Bildung selbständig in die Hand zu nehmen - unter Mißbrauch meines Namens - so hat es keinen Zweck, daß wir ferner ein gemeinsames Leben führen. Gehe deine Wege, und ich werde die meinigen gehen.» So verbrachte der junge Gymnasiast seine Studienjahre in einer Studentenpension.

Die Berufswahl bereitete ihm Mühe! «Was ich studieren sollte, war mir damals noch keineswegs klar. Ich schwankte zwischen Übertritt ins Polytechnikum (Chemie) und dem Studium der Geschichte, der Philosophie und der Medizin. Mein Vater empfahl mir ersteres, meine Neigungen gehörten zum historischen Studium, und die praktische Überlegung trieb mich zum Studium der Medizin als des ökonomisch sichersten Berufes. Von letzterem Studium riet mir aber mein Vater dringend ab, der aus Erfahrung am eigenen Leib die Ärzte außerordentlich gering schätzte. » So ließ sich Constantin gegen den Rat des Vaters an der medizinischen Fakultät immatrikulieren und lebte als Student allein und unabhängig. Nach geglücktem Examen wollte er bei seinem Vater einen Versöhnungsversuch einleiten. Das Resultat war folgendes: «Mein erstes war, die freudige Nachricht über das bestandene Examen meinem Vater, dem ich entfremdet war und der wegen meinen früheren Unbotmäßigkeiten und andern kleinen Verfehlungen mir grollte, zu überbringen. Ich erschien, nachdem ich monatelang ihn nicht besucht hatte, unangemeldet, was ihn bereits in Zorn versetzte, so daß er mich nicht einlassen wollte. Ich setzte aber meinen Besuch bei ihm durch. Der Empfang von seiner Seite war auch danach. Die freundliche Mitteilung meines Examenserfolges machte auf ihn keinen Eindruck. Er erwiderte kühl auf russisch : So bist du Arzt. Nun brauchst du weder zu betteln noch zu stehlen, um leben zu können. Diese Antwort versetzte mich in Wut, und der neue Bruch mit meinem sonst sehr verehrten und geliebten Vater war nun ein vollkommener ...»

Was nun? Eine Stelle zu bekommen war nicht einfach. Monakow versuchte in Zürich eine Privatpraxis zu eröffnen. In sechs Wochen meldete sich kein Patient, deshalb ging er nach München und machte von dort aus in der Not eine Praxisvertretung in Oberammergau. Eine kleine Annonce machte ihm Mut, sich als Schiffsarzt zu melden, und so reiste er nach Brasilien. Während dieser Zeit bemühten sich einige Freunde um ihn, und so konnte er bei seiner Rückkehr am 30. Januar 1878 die Stelle eines Sekundärarztes in St. Pirminsberg (Kanton St. Gallen) annehmen.

In diese Zeit fällt die - für ihn wiederum charakteristische - Erledigung seiner Doktorarbeit.

Das ihm von seinem ehemaligen Chef E. Hitzig vorgeschlagene Disser- 
tationsthema über die «primäre Verrücktheit» zog ihn wenig an. «Trotzdem », so sagt er, «liegt diese Arbeit als fertiges Manuskript in meiner Schublade, ein unreifes Erzeugnis, aber nicht ohne eigene gute Ansätze von Gedanken, die in das hirnphysiologische Gebiet hineingreifen. Das Ganze kam mir aber schon damals wissenschaftlich so armseelig vor, daß ich trotz eifriger Aufmunterung Hitzigs mich nicht entschließen konnte, die Arbeit als Dissertation zu verwerten. Mein Streben nach Zurückstellung dieses Erstlingsopus ging dahin, mich auf Grund hirnpathologischer Beobachtungen bei den Sektionen und am Mikroskop und unter Aufnahme experimenteller Forschung auf dem Gebiet der Hirnphysiologie - und -anatomie möglichst zu orientieren und auf diesem Gebiet etwas Erkleckliches zu leisten. » So berichtet er später: «Ich faßte den Plan Herderkrankungen anatomisch und klinisch möglichst genau zu bearbeiten und die Ergebnisse als Dissertation zu verwerten. Ich verfaßte die Arbeit 1880 und reichte das Manuskript dem Dekanat der medizinischen Fakultät als Dissertation ein. Die Arbeit wurde, obschon sie ohne jede Anregung und Anleitung von seiten eines Professors entstanden war, glatt angenommen, ja, Forel schrieb mir sogar von sich aus einige freundliche Worte.»

In der Folge erscheinen grundlegende Arbeiten auf neuroanatomischem Gebiet, und das Jahr 1885 bildet einen weiteren Markstein im Leben des Forschers: die Habilitation. Als Habilitationsschrift wurde eine schon veröffentlichte Arbeit über die optischen Bahnen und Zentren eingereicht. «Eine ad hoc verfaßte Habilitationsschrift wurde ihm», wie er sagt, «mit Rücksicht auf den unbestrittenen wissenschaftlichen Wert der vorliegenden erlassen. Als Referent wurde Eichноrsт bestellt, Forel war Dekan. Die Habilitation wurde einstimmig zugelassen und die Probevorlesung über die klinische Bedeutung des Patellarreflexes gehalten.» Vor seiner Niederlassung in Zürich suchte er schon von St. Pirminsberg aus eine Stelle. Glücklicherweise - so können wir rückblickend sagen - mißlangen diese Versuche. Er hoffte nämlich, 1882 an die Waldau oder nach Préfargier gewählt zu werden. Préfargier war bald ausgeschaltet, in der Waldau wurde von SPEYR gewählt, ein Schüler Forels, der auf Anfrage Monakow zur Antwort gab : «Er hätte die Verpflichtung gefühlt, in erster Linie für die Zukunft seiner Assistenten zu sorgen. «Diese wiederum charakteristische Episode im Leben Monakows soll der Vollständigkeit wegen auch von bernischer Seite beleuchtet werden. Monakow hatte sich um die Stelle des Sekundärarztes beworben. Aus dem Gerede, das über SchÄrer (damaliger Direktor) als Schwinger und Volksmann zirkulierte, hatte Monakow falsche Schlüsse gezogen. Als er sich dort 
vorstellte, glaubte er, er müsse sich so benehmen, als hätte er eine Rolle im heutigen Berner Heimatschutztheater zu spielen. Er habe den Rock ausgezogen, weil es heiß war, habe sich rittlings auf den Stuhl gesetzt und die Arme auf der Lehne verschränkt und einen Stumpen angezündet. Schärer, der aber nicht nur Schwinger, sondern zuerst Bernerburger war und Lebensart kannte, habe dies nicht recht gefallen, und so entschloß er sich für den andern, wie erwähnt, von Speyr. Acht Jahre später besann sich aber die Berner Fakultät nochmals auf Monakow, um ihn als Direktor nach Bern zu wählen. Obwohl er selbst nichts mehr erwähnt, hat er diesen Ruf abgelehnt. So kam er von St. Pirminsberg nach Zürich, baute sich eine gutgehende Privatpraxis auf und begann seine Vorlesungstätigkeit.

Die Ernennung Monakows zum Professor verdient ebenfalls festgehalten zu werden. «Da die Vorgänge», wie er sagt, "vom menschlichen Standpunkt aus so interessant und für die damaligen Fakultätsverhältnisse so charakteristisch seien, wolle er sie den Lesern nicht vorenthalten.

Im Juli 1894 erhielt er einen Ruf nach Innsbruck und teilte dies der Erziehungsdirektion wie auch dem Dekan der Fakultät (HAAB) mit, der ihm riet die Stelle anzunehmen. Am 19. Juli fand die Fakultätssitzung statt, nach deren Resultat sich Monakow bei einem Kollegen erkundigte. Geheimnisvolles Schweigen, verlegenes Lächeln und der Bescheid, der Dekan würde den Beschluß der Fakultät schriftlich mitteilen. Im Schreiben vom 20. Juli stand, daß die Fakultät die Beförderung ablehne, mit der Argumentation, daß hier nicht diese günstige Stellung geschaffen werden könne, zumal die Schöpfung einer staatlich unterstützten Nervenpoliklinik und Klinik für die andern Privatdozenten einen unter Umständen verhängnisvollen Präzedenzfall zu bilden imstande wäre. Dieses Schreiben empörte Monakow. Der Erziehungsdirektor wies den Beschluß zurück, mit der Begründung, die wissenschaftliche Argumentation fehle. Die Fakultät betonte daraufhin, die Ablehnung beruhe nur auf äußeren Gründen. So beschloß der Erziehungsrat zuhanden des Regierungsrates die Kreierung eines etatmäßigen Extraordinariates für hirnanatomische Fächer und Nervenpoliklinik. Monakow verhehlt auch nicht, daß sowohl der damalige Internist (ЕICHнORST) wie auch der Anatom (SтӧнR) von der Notwendigkeit dieses Lehrstuhls nicht überzeugt waren. Seine Stellung in der Fakultät war eine wenig erfreuliche. So schreibt er: «Mein erster Besuch der Fakultätssitzung war bereits für mich und die übrigen Anwesenden gleich peinlich. Prof. Ribbert las nämlich als Aktuar in extenso das Protokoll der vorhergehenden Sitzung in welcher alle mir feindlich gesinnten oder ablehnenden .Voten niedergelegt waren. In gu- 
ten Beziehungen stand ich zu wenigen. Sobald ich einen begründeten Antrag oder irgendeine Anregung in einer Sitzung einbrachte, da vereinigten sich fast alle gegen mich und mitunter mit einer fast verletzenden Überlegenheit (wir sind Ordinarii). Das Dekanat wurde mir nie angeboten, nur als Protokollführer wurde ich gewählt, ich lehnte aber nach kurzer Wirksamkeit dieses Ehrenamt ab (wegen Graphospasmus).

Die wissenschaftliche Arbeit ging intensiv weiter, Schüler aus aller Welt wollten mit ihm und unter ihm arbeiten. Er wurde aufgefordert, an internationalen Tagungen Hauptreferate zu halten, und war als Mitglied der Braincommission (internationale Vereinigung der Hirnforscher) Ehrengast bei der hundertjährigen Stiftungsfeier der k. k. wissenschaftlichen Akademie in Wien. Die wissenschaftliche Welt war zusammengekommen, und vor Beginn der festlichen Sitzung wurden die Ehrengäste dem Erzherzog vorgestellt. Monakow kam als zehnter an die Reihe und konnte eine Ansprache des Erzherzogs an den englischen Gelehrten mitanhören. «Sie war so ergötzlich, ebenso wie die einige Minuten später an mich gerichtete», führte er aus, «daß sie ihres, geistreichen" Inhaltes wegen wörtlich wiedergegeben wird.

Zum englischen Gelehrten:

Erzherzog: Sie kommen weit her. Ich habe mein Englisch längst verschwitzt.

Englischer Gelehrter (verlegen): Oh, wie. Switzt. Switzt, was ist switzt.

Erzherzog: Ich habe das Englische längst vergessen.

Englischer Gelehrter: Oh, oh, verstehe (verneigt sich mit ehrwürdigem Lächeln). - Fertig.

Meine Begrüßung:

Erzherzog: Sie kommen aus der Schweiz.

Ich: Jawohl, zu dienen, Hoheit.

Erzherzog: Gibt es viele Akademien in der Schweiz?

Ich: Wir besitzen leider nicht eine einzige.

Erzherzog (erstaunt und ernst): Ja, wie kommen Sie denn dann hieher?

Ich: Ja, ich bin Mitglied der Braincommission, einer wissenschaftlichen

Unterkommission der internationalen Vereinigung von Hirnforschern.

Erzherzog: (beruhigt und freundlich): So, so, dann beschäftigen Sie sich mit Hirnforschung. Nun, das ist sehr schön und nützlich, heutzutage, wo alle Menschen neurasthenisch sind, ha, ha, ha!»

«Ein etwas müder Wink», so fährt Monakow fort, «und ich war entlassen. Und so ging es weiter ca. 20 Minuten, während der dicht gefüllte Saal unge- 
duldig auf die Eröffnung der Sitzung wartete und die Zuschauer ihre Glossen machten über den vor ihren Augen abgehaltenen erzherzoglichen Cercle. Die ,geistreichen' Begrüßungen des Erzherzogs standen in solchem Gegensatz zu dem sonstigen Inhalt und Gesprächsniveau des Abends und zu der Qualität des übrigen Publikums, daß sie sich mir tief einprägten. Nun hatte ich meine Belehrung, wie ungefähr die Konversation in den Hofkreisen in üblicher Weise gepflegt wird.»

Diese so offene und kritische Schilderung fand nicht überall Zustimmung. So schrieb ihm der Neurologe WinkLer aus Utrecht (in seinem besonderen Deutsch!), der die Vita mea zu lesen bekam: «Den Unsinn vom Erzherzog würde ich nicht drucken lassen. Es ist schon außerordentlich großes, welches der greise Erzherzog leistet, durch die Tat zu einer Festsitzung zu kommen. Er kann es nicht helfen, daß er dumm ist und jedem etwas Freundliches sagen muß. An unserer Königin bin ich vier- oder fünfmal vorgestellt worden in verschiedenen Qualitäten. Als Dekan der Fakultät, als Rector magni ficus in Amsterdam etc. Jedesmal hat sie mich gefragt: ,Wie lange dauern die Sommerferien ?' Unsere Königin ist sehr gescheit, aber über alles mit jeder Person etwas Vernünftiges sagen zu können, dazu gehört eine gewaltige Virtuosität ...»

Es braucht abschließend nicht besonders hervorgehoben zu werden, daß mit dieser Schilderung ein unvollständiges Bild dieses Neurologen entstanden ist.

Die medizinisch-wissenschaftliche Seite wurdenicht berücksichtigt, die Experimente neuroanatomischer- und neurophysiologischer Natur, die vor allem in der St. Pirminsbergerzeit zu neuen Entdeckungen führten, wurden nicht erwähnt.

Die Absicht dieser Ausführungen war, Interesse zu wecken für eine Persönlichkeit, die getragen von einer universalen Bildung, durch eine gründliche naturwissenschaftliche Schulung zur Neurologie kam. In seinem Fach und in seinem Rahmen baute er sich eine Stellung auf, behauptete und verteidigte sie gegenüber einer Umwelt, die ihn einerseits verehrte und schätzte und anderseits durch Nichtbeachtung seiner Fähigkeiten liegenließ.

\section{Literaturhinweise}

Minkowski M., 50 Jahre Schweizerische Neurologische Gesellschaft, Archiv für Neurologie und Psychiatrie 82, 1/2.

WYRSCH JАKоB, Hundert Jahre Waldau, Hans Huber, Bern. 